\title{
Non-Oberbeck-Boussinesq Effects in Gaseous Rayleigh-Bénard Convection
}

\author{
Guenter Ahlers, ${ }^{1}$ Francisco Fontenele Araujo, ${ }^{2}$ Denis Funfschilling, ${ }^{1}$ Siegfried Grossmann, ${ }^{3}$ and Detlef Lohse ${ }^{2}$ \\ ${ }^{1}$ Department of Physics and iQCD, University of California, Santa Barbara, California 93106, USA \\ ${ }^{2}$ Department of Applied Physics and J.M. Burgers Centre for Fluid Dynamics, University of Twente, \\ 7500 AE Enschede, The Netherlands \\ ${ }^{3}$ Department of Physics, Philipps-University of Marburg, Renthof 6, D-35032 Marburg, Germany
}

(Received 2 December 2006; published 29 January 2007)

\begin{abstract}
Non-Oberbeck-Boussinesq (NOB) effects are measured experimentally and calculated theoretically for strongly turbulent Rayleigh-Bénard convection of ethane gas under pressure where the material properties strongly depend on the temperature. Relative to the Oberbeck-Boussinesq case we find a decrease of the central temperature as compared to the arithmetic mean of the top- and bottom-plate temperature and an increase of the Nusselt number. Both effects are of opposite sign and greater magnitude than those for NOB convection in liquids like water.
\end{abstract}

DOI: 10.1103/PhysRevLett.98.054501

Turbulent convection in a fluid heated from below and cooled from above (Rayleigh-Bénard convection) is an important model system in fluid dynamics [1]. The induced temperature difference $\Delta$ across a sample is represented by the Rayleigh number $\mathrm{Ra}=\beta g L^{3} \Delta /(\kappa \nu)$ ( $\beta$ is the thermal expansion coefficient, $g$ the acceleration of gravity, $L$ the sample height, $\kappa$ the thermal diffusivity, and $\nu$ the kinematic viscosity). The problem usually is analyzed within an approximation due to Oberbeck [2] and Boussinesq [3] $(\mathrm{OB})$, where it is assumed that all fluid properties are constant within the entire sample except for the density where it induces the buoyancy force. Here we address, both experimentally and theoretically, the nature of deviations from this approximation.

A central aspect of this system is an understanding of the boundary layers (BLs) near the top and bottom plates. At modest Ra they remain laminar while the fluid interior is turbulent, and their instabilities impact the Nusselt number $\mathrm{Nu}$ (the effective thermal conductivity $\Lambda_{\text {eff }}$ normalized by the diffusive thermal conductivity $\Lambda$ ) [4,5]. At much larger $\mathrm{Ra}$ it was suggested [6-8] that the BLs become turbulent as well, that they then no longer influence $\mathrm{Nu}$, and that an asymptotic ("ultimate" or "Kraichnan") regime is achieved where $\mathrm{Nu} \sim \mathrm{Ra}^{1 / 2}$. In the present Letter we show, by comparing theoretical calculations with new experimental measurements, that the laminar BLs are also significantly influenced by non-Oberbeck-Boussinesq (NOB) effects. These effects can be described well by an extension of the Prandtl-Blasius boundary-layer theory $[9,10]$.

Though NOB effects in turbulent Rayleigh-Bénard convection were measured already 15 years ago [11,12], a quantitative comparison between $\mathrm{OB}$ and NOB convection was only done recently [13]. However, such study was restricted to NOB effects in liquids like water and glycerol. It found a decrease of $\mathrm{Nu}$ and an increase of the center temperature $T_{c}$ as compared to the $\mathrm{OB}$ case. The latter could be explained quantitatively by an extension of the Prandtl-Blasius BL theory [13]. Extending this theory to
PACS numbers: 47.27.-i, 47.20.Bp

gases is considerably more challenging, as then also the density depends on temperature (beyond the OB dependence), leading to a density boundary layer. Moreover, all material properties such as $\Lambda, \beta$, the shear viscosity $\eta$, and the specific heat $c_{P}$ depend on both temperature and density. Here we shall show that nevertheless an extension of the Prandtl-Blasius BL theory is possible.

For ethane gas close to its critical point both our theory and experiment show that the NOB effects on $T_{c}$ are much larger and opposite to those for liquids: we observe a considerable decrease of $T_{c}$ as compared to the OB case. Experiment moreover reveals that also the effect on $\mathrm{Nu}$ is stronger and opposite to that in liquids: $\mathrm{Nu}_{\mathrm{NOB}}$ is larger than $\mathrm{Nu}_{\mathrm{OB}}$.

The experiments were done with a cylindrical sample of height $L=7.62 \mathrm{~cm}$ and aspect ratio $\Gamma \equiv L / D=0.999 \pm$ 0.002 ( $D$ is the sample diameter). The cell had copper top and bottom plates and a high-tensile-strength steel side wall. We used various values of $\Delta \equiv T_{b}-T_{t}\left(T_{b}\right.$ and $T_{t}$ are the bottom- and top-plate temperatures, respectively) at a mean temperature $T_{m} \equiv\left(T_{b}+T_{t}\right) / 2=39.98^{\circ} \mathrm{C}$. Measurements were made at various pressures $P_{m}$ that were held constant to $\pm 1 \mathrm{mBar}$. The temperature $T_{t}$ was always kept larger than the condensation temperature or the temperature corresponding to the critical isochore (see Fig. 1). For a given $P_{m}$ the values of $\Delta$ then determined Ra, which was evaluated from properties at $T_{m}$. The center temperature $T_{c}$ was deduced from eight thermometers at uniformly distributed azimuthal locations on the outside of the sidewall at half height of the cell; see Refs. [5,14] for details.

The results for $T_{c}-T_{m}$ as a function of $\Delta$ for various $P_{m}$ are presented in Fig. 2(a). Clearly, in all cases the center temperature is smaller than the arithmetic mean $T_{m}$ of $T_{b}$ and $T_{t}$, signaling a breaking of the top-bottom symmetry. Assuming that the time-averaged temperature in the bulk is constant with height, i.e., that the sum $\Delta_{b}+\Delta_{t}$ of the temperature drops over the bottom and the top BL equals $\Delta$, this result can be expressed as $\chi=\Delta_{b} / \Delta_{t}$, see Fig. 2(b). Deviations from $\chi=1$ signal NOB effects on 


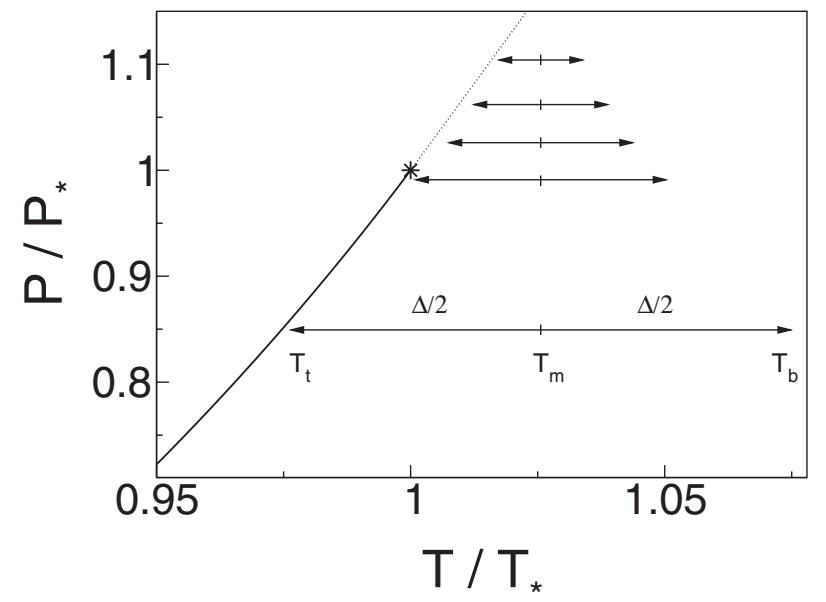

FIG. 1. Pressure-temperature plane in reduced units. Star: critical point of ethane $\left(T_{*}=32.18{ }^{\circ} \mathrm{C}, P_{*}=48.718\right.$ bars $)$. Heavy line: liquid-vapor coexistence curve. Dotted line: critical isochore. The horizontal arrows show the maximum temperature intervals $\Delta$, centered about $T_{m}=39.98^{\circ} \mathrm{C}$, that were used at each of the pressures $P_{m} / P_{*}=0.849,0.991,1.026,1.062$, and 1.104 (bottom to top).

$T_{c}$. Note that in the present case for ethane we not only have $\chi>1$ as compared to $\chi<1$ for liquids, but also that the modulus of the relative deviation from one is much larger than in the water case; i.e., NOB effects on the center temperature are opposite and much stronger in ethane as compared to the liquids analyzed in Ref. [13].

The lines shown in Fig. 2 are obtained by an extension of the BL theory of Ref. [13], which now involves the density field $\rho$ in addition to those of temperature $T$ and velocity $\mathbf{u}$. In the spirit of Prandtl-Blasius theory, such fields are assumed to be stationary and only dependent on the longitudinal $x$ and the transversal $z$ coordinates. As shown below, this kind of $\mathrm{BL}$ approach reasonably describes $\rho(x, z), T(x, z), u_{x}(x, z)$, and $u_{z}(x, z)$.

In Prandtl's approximation, mass and momentum conservation can be expressed as:

$$
\begin{gathered}
\partial_{x}\left\{\rho u_{x}\right\}+\partial_{z}\left\{\rho u_{z}\right\}=0, \\
\rho\left\{u_{x} \partial_{x} u_{x}+u_{z} \partial_{z} u_{x}\right\}=\partial_{z}\left\{\eta \partial_{z} u_{x}\right\},
\end{gathered}
$$

with the boundary conditions $u_{x}(x, 0)=0, u_{z}(x, 0)=0$, and $u_{x}(x, \infty)=U_{c}$. Here, $U_{c}$ is the typical large-scale wind velocity [8]. From energy conservation, one can derive the following equation for the temperature field $[15,16]$ :

$$
u_{x} \partial_{x} T+u_{z} \partial_{z} T+\frac{\gamma-1}{\beta}\left\{\partial_{x} u_{x}+\partial_{z} u_{z}\right\}=\frac{\gamma}{\rho c_{P}} \partial_{z}\left\{\Lambda \partial_{z} T\right\},
$$

where $\gamma=c_{P} / c_{V}$ and the boundary conditions are $T(x, 0)=T_{b, t}, T(x, \infty)=T_{c}$. Just as in Ref. [13], the BLs are coupled by the condition that the heat fluxes $Q_{b, t}$ through the top and bottom BLs must be equal:

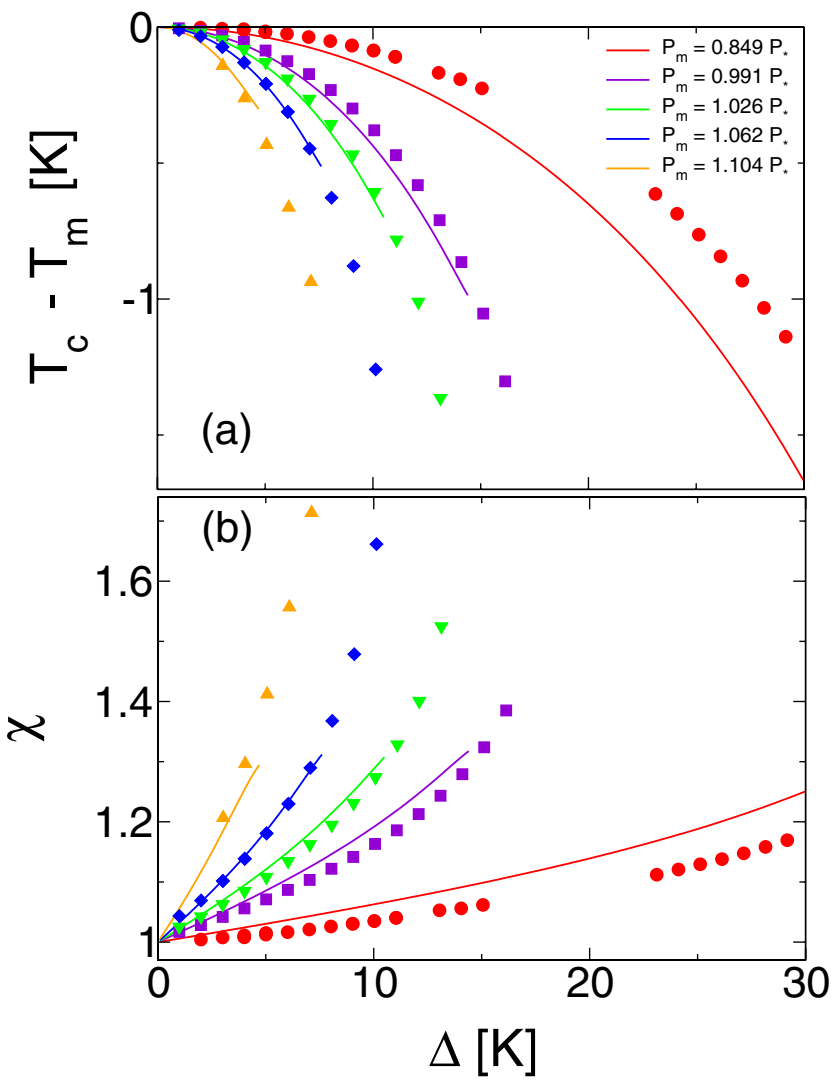

FIG. 2 (color online). (a) Deviation $T_{c}-T_{m}$ of the center temperature $T_{c}$ from $T_{m}$ as function of $\Delta$ and (b) resulting ratio $\chi=\Delta_{b} / \Delta_{t}$ of the temperature drops across the bottom and the top boundary layers. Symbols: experiment. Lines: theory. As in Fig. 1, the pressure $P_{m}$ ranges from $0.849 P_{*}$ to $1.104 P_{*}$.

$$
Q_{b}=-\left.\Lambda_{b} \partial_{z} T\right|_{b}=-\left.\Lambda_{t} \partial_{z} T\right|_{t}=Q_{t} .
$$

Equations (1)-(4) with the corresponding boundary conditions must be solved self-consistently. The dependences of the material properties such as $\eta, \Lambda, c_{P}$, and $\beta$ on temperature and density are taken from Ref. [17], see Fig. 3.

As in Prandtl's theory, the system of partial differential equations (1)-(3) can be reduced to ordinary differential equations (ODEs) through a stream function $\Psi$ :

$$
\tilde{\rho} u_{x}=\partial_{z} \Psi, \quad \tilde{\rho} u_{z}=-\partial_{x} \Psi,
$$

where $\tilde{\rho} \equiv \rho / \rho_{m}$ is the density nondimensionalized with $\rho_{m}=\rho\left(T_{m}, P_{m}\right)$. Since the continuity equation is automatically fulfilled by (5), we may introduce a selfsimilarity variable $\tilde{z} \equiv z / \ell_{c}(x)$ and a similarity function $\tilde{\Psi}(\tilde{z})=\Psi(x, z) /\left(\ell_{c} U_{c}\right)$, such that $\ell_{c}(x)=\sqrt{x \nu_{m} / U_{c}}$. Thus, the velocity components are

$$
u_{x}=U_{c} \frac{\tilde{\Psi}^{\prime}}{\tilde{\rho}}, \quad u_{z}=\frac{\nu_{m}}{2 \ell_{c}}\left\{\tilde{z} \frac{\tilde{\Psi}^{\prime}}{\tilde{\rho}}-\frac{\tilde{\Psi}}{\tilde{\rho}}\right\}
$$

with boundary conditions $\tilde{\Psi}(0)=0=\tilde{\Psi}^{\prime}(0)$ and $\tilde{\Psi}^{\prime}(\infty)=\tilde{\rho}_{c}$. 

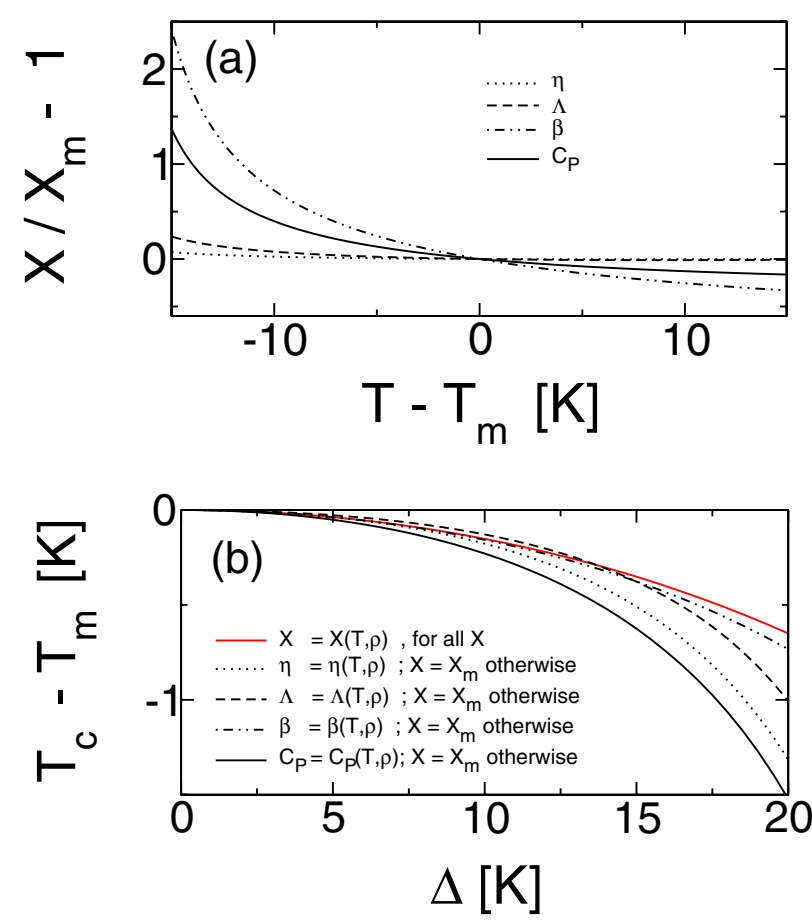

FIG. 3 (color online). (a) Relative deviations $\left(X-X_{m}\right) / X_{m}$ of ethane properties from their values $X_{m}$ at $T_{m}=40{ }^{\circ} \mathrm{C}$ and $P_{m}=$ $0.849 P_{*}$. The curves are based on Ref. [17]. (b) Predicted $T_{c}-$ $T_{m}$ for hypothetical gases, with one of the material parameters varying as function of temperature and all others fixed at their values $X_{m}$ at $T_{m}=40^{\circ} \mathrm{C}$ and $P_{m}=0.849 P_{*}$.

In terms of (6), the viscous BL equation (2) can be written as:

$$
\begin{aligned}
\tilde{\eta} & \tilde{\Psi}^{\prime \prime \prime}+\left\{\frac{1}{2} \tilde{\Psi}+\tilde{\eta}^{\prime}-2 \frac{\tilde{\rho}^{\prime}}{\tilde{\rho}} \tilde{\eta}\right\} \tilde{\Psi}^{\prime \prime} \\
& +\left\{-\frac{1}{2} \frac{\tilde{\rho}^{\prime}}{\tilde{\rho}} \tilde{\Psi}+\left[2\left(\frac{\tilde{\rho}^{\prime}}{\tilde{\rho}}\right)^{2}-\frac{\tilde{\rho}^{\prime \prime}}{\tilde{\rho}}\right] \tilde{\eta}-\frac{\tilde{\rho}^{\prime}}{\tilde{\rho}} \tilde{\eta}^{\prime}\right\} \tilde{\Psi}^{\prime}=0,
\end{aligned}
$$

where $\tilde{\eta} \equiv \eta / \eta_{m}$ is the dimensionless viscosity, $\tilde{\eta}^{\prime}=$ $\left(\frac{\partial \tilde{\eta}}{\partial \tilde{\Theta}}\right)_{\tilde{\rho}} \tilde{\Theta}^{\prime}+\left(\frac{\partial \tilde{\eta}}{\partial \tilde{\rho}}\right)_{\tilde{\Theta}} \tilde{\rho}^{\prime}$ the corresponding gradient, and $\tilde{\Theta} \equiv$ $\left(T-T_{t}\right) / \Delta$ the dimensionless temperature.

Next, assuming that the pressure is constant at $P_{m}$ throughout the fluid, the equation of state leads to [15]:

$$
\tilde{\rho}^{\prime}=-\tilde{\rho} \tilde{\beta} \tilde{\Theta}^{\prime},
$$

with $\tilde{\beta} \equiv \beta \Delta$. Here, the boundary conditions are $\tilde{\rho}(0)=$ $\tilde{\rho}_{b, t}, \tilde{\rho}^{\prime}(0)=-\tilde{\rho}_{b, t} \tilde{\beta}_{b, t} \tilde{\Theta}_{b, t}^{\prime}$, and $\tilde{\rho}(\infty)=\tilde{\rho}_{c}$.

Finally, we also write the temperature Eq. (3) in selfsimilar form as [15]:

$$
\tilde{\Lambda} \tilde{\Theta}^{\prime \prime}+\left\{\frac{1}{2} \tilde{c}_{P} \operatorname{Pr} \tilde{\Psi}+\tilde{\Lambda}^{\prime}\right\} \tilde{\Theta}^{\prime}=0,
$$

where $\tilde{\Lambda} \equiv \Lambda / \Lambda_{m}$ and $\tilde{c}_{P} \equiv c_{P} / c_{P, m}$. Equation (9) is subject to $\tilde{\Theta}(0)=\tilde{\Theta}_{b, t}$ and $\tilde{\Theta}(\infty)=\tilde{\Theta}_{c}$.

The three coupled ODEs (7)-(9) with the respective boundary conditions and the heat-flux conservation (4) are solved numerically with a shooting method [15]. The integration domain is defined with the same values of $T_{m}$ and $P_{m}$ as those used in the experiments, but restricted to $\Delta$ ranges where the material properties are convex. There are no free fit parameters. As shown in Fig. 2, the agreement between theory and experiment is reasonable, in particular, for the pressures larger than $P_{*}$. Further theoretical results, such as temperature and density profiles, will be presented elsewhere [15].

What material property is the main origin of the decreased $T_{c}$ ? To find out, we recalculated $T_{c}$ for various hypothetical gases, with all material properties constant as in the $\mathrm{OB}$ case and evaluated at $T_{m}=40^{\circ} \mathrm{C}$ except for one. The one temperature-dependent property has the same temperature dependence as ethane at $P / P_{*}=0.849$. The result is shown in Fig. 3(b). Remarkably, every individual temperature dependence is already strong enough to produce a NOB deviation of $T_{c}$ of a size that is comparable to the full NOB effect, reflecting the complicated nonlinear dependence of $T_{c}$ on the material properties and the nonlinear dependence of the material properties on temperature [Fig. 3(a)]. However, we think that the centertemperature reduction observed here is a more general feature for gases as compared to the center-temperature increase in liquids [13]: in liquids $(\partial \eta / \partial T)_{\rho}<0$ leads to a thinner bottom viscous $\mathrm{BL}$ in the $\mathrm{NOB}$ case as compared to the $\mathrm{OB}$ case. This thinner bottom viscous BL induces also a thinner thermal BL between the center and the hot bottom plate, implying an enhanced center temperature. For gases, however, the bottom viscous BL expands due to the temperature dependence of the density, inducing also an extension of the thermal BL between the hot bottom and the center, thus implying a larger temperature drop across that BL, i.e., a reduced center temperature.

At present we do not have a theory for NOB effects on $\mathrm{Nu}$. However, we show some experimental results in Fig. 4(a) as a function of $\mathrm{Ra}$ on logarithmic scales. In addition, a large number of data points with relatively small $\Delta$ and $\beta \Delta \leq 0.05$ were obtained over a wide range of $T_{m}$ and $P_{m}$. They served as an experimental estimate of $\mathrm{Nu}_{\mathrm{OB}}$ that could be represented well by $\mathrm{Nu}_{\mathrm{OB}}=$ $0.1826 \mathrm{Ra}^{0.292}$ [18]. This is shown as a dotted line in Fig. 4(a). Particularly at the highest pressure one sees substantial deviations of the data from the dotted line, which we interpret as strong NOB effects. The deviations become more clear in Fig. 4(b), where the ratio $\mathrm{Nu}_{\mathrm{NOB}} / \mathrm{Nu}_{\mathrm{OB}}$ is shown. In contrast to the measurements for water [13], where NOB effects caused a decrease, albeit by only a percent or so, one sees in Fig. 4(b) that NOB effects increase Nu by as much as $20 \%$ in the ethane case.

Although the temperature dependence of all material properties seems to contribute significantly to the NOB effects, it is of interest to see whether one of them could be chosen as a surrogate to represent the effect of all in an 

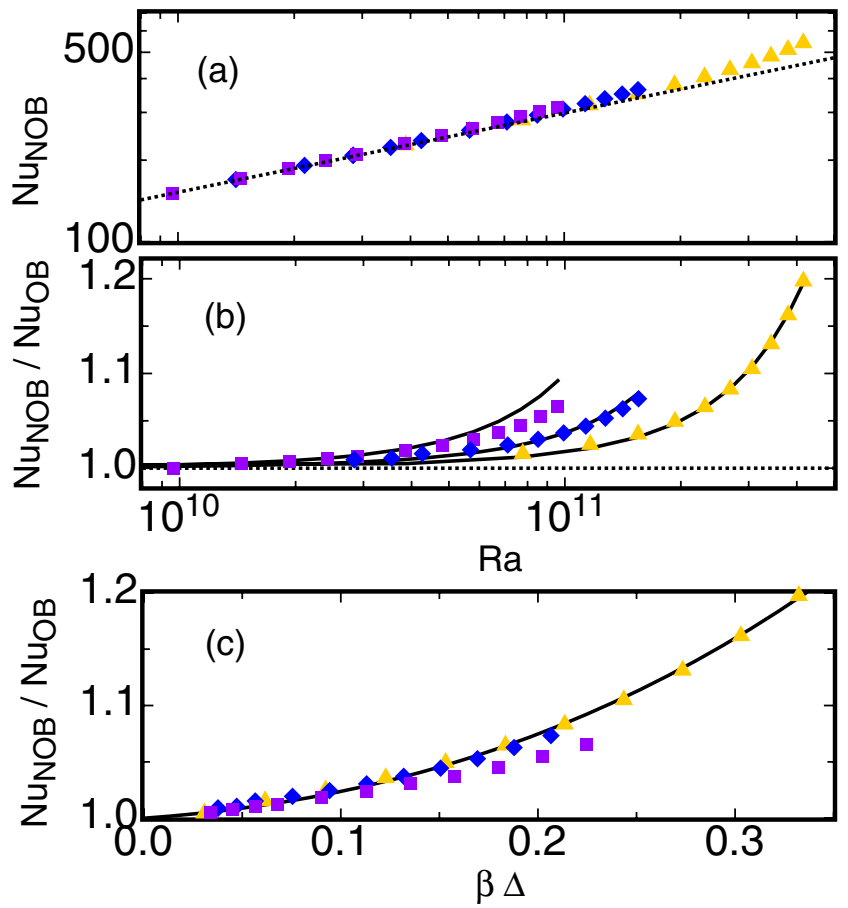

FIG. 4 (color online). (a) $\mathrm{Nu}_{\mathrm{NOB}}$ and (b) $\mathrm{Nu}_{\mathrm{NOB}} / \mathrm{Nu}_{\mathrm{OB}}$ as function of Ra for $P / P_{*}=1.104$ (yellow triangles), 1.062 (blue diamonds), and 0.991 (purple squares). (c) $\mathrm{Nu}_{\mathrm{NOB}} / \mathrm{Nu}_{\mathrm{OB}}$ as a function of $\beta \Delta$. Dotted lines: $\mathrm{Nu}_{\mathrm{OB}}=0.1826 \mathrm{Ra}^{0.292}$. Solid lines: $\mathrm{Nu}_{\mathrm{NOB}} / \mathrm{Nu}_{\mathrm{OB}}=1+0.125(\beta \Delta)+1.012(\beta \Delta)^{2}+1.150(\beta \Delta)^{3}$.

empirical manner. To this end, we plotted the data as a function of $\beta \Delta$ in Fig. 4(c). One sees that the three data sets are nearly, but not completely, collapsed onto a single curve that can be represented by $\mathrm{Nu}_{\mathrm{NOB}} / \mathrm{Nu}_{\mathrm{OB}}=$ $1+0.125(\beta \Delta)+1.012(\beta \Delta)^{2}+1.150(\beta \Delta)^{3}$. This representation is shown as solid lines in both Figs. 4(b) and 4(c). Although not a perfect fit, it does seem to serve as a useful estimate of the approximate size of NOB effects on $\mathrm{Nu}$ in gases. From this analysis we conclude, for instance, that $\mathrm{Nu}_{\mathrm{NOB}}$ will differ from $\mathrm{Nu}_{\mathrm{OB}}$ by about $3 \%$ or less when $\beta \Delta$ is about 0.1 or less.

In this Letter we presented experimental data and theoretical calculations based on an extension of the PrandtlBlasius boundary-layer theory, for the center temperature $T_{c}$ of a turbulent Rayleigh-Bénard sample under nonOberbeck-Boussinesq (NOB) conditions. Theory and experiment agree remarkably well without the need for any adjustable parameters. We also presented new measurements of the Nusselt number Nu under NOB conditions and found strong positive deviations from the $\mathrm{OB}$ case. The deviations could be represented empirically by a simple function of the relative temperature difference $\beta \Delta$ across the sample. This function makes it possible to estimate approximately the size of deviations from the OB case for other data. In particular, it suggests that the relatively rapid rise of Nu with Ra observed by Chavanne et al. [19] (which was interpreted by those authors as evidence for the ulti- mate regime) probably cannot be attributed to NOB effects.

The work at Santa Barbara was supported by the US National Science Foundation through Grant No. DMR0243336. This work is part of the research program of the Stichting voor Fundamenteel Onderzoek der Materie (FOM), which is financially supported by the Nederlandse Organisatie voor Wetenschappelijk Onderzoek (NWO).

[1] See, for instance, L. P. Kadanoff, Phys. Today 54, No. 8, 34 (2001).

[2] A. Oberbeck, Ann. Phys. Chem. (Berlin) 7, 271 (1879).

[3] J. Boussinesq, Theorie Analytique de la Chaleur (Gauthier-Villars, Paris, 1903), Vol. 2.

[4] S. Ciliberto, S. Cioni, and C. Laroche, Phys. Rev. E 54, R5901 (1996).

[5] G. Ahlers, E. Brown, and A. Nikolaenko, J. Fluid Mech. 557, 347 (2006).

[6] R. H. Kraichnan, Phys. Fluids 5, 1374 (1962).

[7] E. A. Spiegel, Annu. Rev. Astron. Astrophys. 9, 323 (1971).

[8] S. Grossmann and D. Lohse, J. Fluid Mech. 407, 27 (2000); Phys. Rev. Lett. 86, 3316 (2001); Phys. Rev. E 66, 016305 (2002); Phys. Fluids 16, 4462 (2004).

[9] H. Schlichting and K. Gersten, Boundary Layer Theory (Springer-Verlag, Berlin, 2000), 8th ed.

[10] K. Stewartson, The Theory of Laminar Boundary Layers in Compressible Fluids (Oxford University Press, New York, 1964).

[11] X.Z. Wu and A. Libchaber, Phys. Rev. A 43, 2833 (1991).

[12] J. Zhang, S. Childress, and A. Libchaber, Phys. Fluids 9, 1034 (1997).

[13] G. Ahlers, E. Brown, F. Fontenele Araujo, D. Funfschilling, S. Grossmann, and D. Lohse, J. Fluid Mech. 569, 409 (2006).

[14] E. Brown, D. Funfschilling, A. Nikolaenko, and G. Ahlers, Phys. Fluids 17, 075108 (2005).

[15] F. Fontenele Araujo, S. Grossmann, and D. Lohse, Phys. Rev. E (to be published).

[16] L. D. Landau and E. M. Lifshitz, Fluid Mechanics (Pergamon, New York, 1987).

[17] D. G. Friend, H. Ingham, and J. F. Ely, J. Phys. Chem. Ref. Data 20, 275 (1991).

[18] We note that this result may be affected, to the extent of a few percent, by the nonlinear influence of the sidewall on the heat transport [20,21]; only the conductance of the empty cell was subtracted. However, the sidewall should not affect $\mathrm{Nu}_{\mathrm{NOB}} / \mathrm{Nu}_{\mathrm{OB}}$ very much because its influence will, to a very large extent, cancel in the ratio.

[19] X. Chavanne, F. Chilla, B. Castaing, B. Hebral, B. Chabaud, and J. Chaussy, Phys. Rev. Lett. 79, 3648 (1997).

[20] G. Ahlers, Phys. Rev. E 63, 015303 (2000).

[21] P. Roche, B. Castaing, B. Chabaud, B. Hebral, and J. Sommeria, Eur. Phys. J. B 24, 405 (2001). 